\title{
TIME-EVOLUTION OF A CAUSTIC
}

\author{
NASIT ARI and ARTHUR D. GORMAN \\ Department of Engineering Science \\ Lafayette College \\ Baston, Pennsylvania 18042 U.S.A. \\ (Received Maxch 26, 1987)
}

ABSTRACT. The Lagrange manifold formalism is adapted to study the time-evolution of caustics associated with high frequency wave propasation in media with both spatial and temporal inhomogeneities.

KEYWORDS AND PHRASES: Wave Propagation, Lagrange manifold, caustics, turning points.

1980 MATHFMATICS SUBJBCT CLASSIFICATION CODE; 34B20

\section{INTHODUCTION}

The utility of the asymptotic series, or seometrical optics, approach developed by Keller [1] and his students [2] for studyins wave-type linear partial differential equations is well known. For example, applied to a differential equation of the form

$\nabla^{2} \psi(\bar{r}, t)-f(\bar{r}, t) \frac{\partial^{2} \psi(\bar{r}, t)}{\partial t^{2}}-\lambda^{2} g(\bar{r}, t) \psi(\bar{r}, t)=0$

where, for definiteness, $(\bar{r}, t)$ is the wave function, $\bar{r}$ refers to the spatial coordinates, $t$ is the time and $\lambda$ is a large parameter, a solution of the form

$\varphi(\overline{\mathbf{r}}, t)=\exp [i \lambda S(\overline{\mathbf{r}}, t)] A(\overline{\mathbf{r}}, t, \lambda)$,

where

$A(\bar{r}, t, \lambda)=\sum_{k=0} A_{k}(\bar{r}, t)(i \lambda)^{-k}, A_{-k}=0$

is assumed. $S(\bar{r}, t)$ may be regarded as a phase and $A(\bar{r}, t, \lambda)$ as an amplitude. Then substituting Equation (1.2) into Equation (1.1) followed by a re-grouping in powers of in leads to 


$$
\begin{aligned}
& \left\{(i \lambda)^{2}\left[(\nabla S)^{2}-f(\bar{r}, t)\left[\frac{\partial S}{\partial t}\right]^{2}+g(\bar{r}, t)\right]+\right. \\
& (i \lambda)\left[\nabla^{2} S-f(\bar{r}, t) \frac{\partial^{2} S}{\partial t^{2}}+2 \nabla S \cdot \nabla-2 f(\bar{r}, t) \frac{\partial S}{\partial t} \frac{\partial}{\partial t}\right] \\
& \left.(i \lambda)^{\circ}\left[\nabla^{2}-f(\bar{r}, t) \frac{\partial^{2}}{\partial t^{2}}\right]\right\} \sum_{k=0} A_{k}(\bar{r}, t)(i \lambda)^{-k}=0 .
\end{aligned}
$$

Then by introducing the wave number and frequency

$$
\overline{\mathbf{p}}=\nabla \mathrm{s}, \quad \omega=-\frac{\mathrm{as}}{\partial \overline{\mathrm{t}}},
$$

respectively, the coefficient of the $(i \lambda)^{2}$ term may be regarded as a Hamiltanian

$$
H=\bar{p} \cdot \bar{p}-f(\bar{r}, t) \omega^{2}+g(\bar{r}, t) .
$$

The standard approach for obtaining the phase involves the intmduction of Hamilton's equations

$$
\begin{array}{ll}
\frac{d \bar{r}}{d r}=\nabla_{p} H & \frac{d \bar{p}}{d r}=-\nabla_{r} H \\
\frac{d t}{d r}=-\frac{\partial H}{\partial \omega} & \frac{d \omega}{d r}=\frac{\partial H}{\partial t}
\end{array}
$$

which leads to the ray trajectories (map)

$$
\begin{array}{ll}
\overline{\mathbf{r}}=\overline{\mathbf{r}}(r, \bar{\sigma}) & \overline{\mathbf{p}}=\overline{\mathrm{p}}(r, \bar{\sigma}) \\
\mathrm{t}=t(r, \bar{\sigma}) & \omega=\omega(r, \bar{\sigma}),
\end{array}
$$

where $r$ is the ray-path parameter and $\bar{\sigma}$ a parameterized initial condition. But at those space-time points where the coordinate space map becomes singular i.e., on the caustic curve where

$$
\operatorname{det}\left[\frac{\partial(\bar{r})}{\partial(\bar{p})}\right]=0
$$

with $\bar{\mu}=(\gamma, \bar{\sigma})$, the geometrical optics procecture cannot be applied directly.

Such difficulties at caustics can often be circumented by using the Lasrange Manifold formalisa of Maslov [3] and Arnold [4], which has recently been extended to determine a class of asymptotic solutions [5] for phenomena modelled by Equation (1.1). Here present a variation of this extension which enables a modelling of the time evolution of the caustic. This algorith also leads to determination of the field on the caustic; but because so much of this aspect of the procedure in [5] applies directly, for brevity we emphasize only those aspects pertinent to modelling the evolution of the caustic. For clarity, we consider the scalar wave equation given in Bquation (1.1), although the analogous vector wave equation could also have been considered. An example is included to illustrate the procedure.

\section{FORALISY}

We assume that near caustics Equation (1.1) has an asymptotic solution of the form

$$
Y(\bar{r}, t)-\int A(\bar{r}, \bar{p}, t, \lambda) \exp \{i \lambda(\bar{r} \cdot \bar{p}-S(\bar{p}, t))] d \bar{p}=O\left(\lambda^{-\infty}\right) \text {. }
$$


The amplitude $A(\bar{r}, \bar{p}, t, \lambda)$ and its derivatives are assumed bounded and $\bar{r} \cdot \bar{p}-S(\bar{p}, t)$ is regarded as a phase, i.e.,

$$
\phi(\bar{r}, \bar{p}, t)=\bar{r} \cdot \bar{p}-s(\bar{p}, t) \text {. }
$$

Carrying the differentiation in Equation (1.1) across the integral in Equation (2.1) leads to

$$
\begin{aligned}
& \int d \bar{p} \exp \{i \lambda \downarrow\}\left\{(i \lambda)^{2}\left[\bar{p} \cdot \bar{p}-f(\bar{r}, t) \omega^{2}+g(\bar{r}, t)\right]+\right. \\
& \left.\quad i \lambda\left(2 \bar{p} \cdot \nabla_{r} A+2 \omega f(\bar{r}, t) \frac{\partial A}{\partial t}\right]+(i \lambda) \circ\left[\nabla_{r}^{2} A-f(\bar{r}, t) \frac{\partial^{2} A}{\partial t^{2}}\right]\right)=0\left(\lambda^{-\infty}\right)
\end{aligned}
$$

Where, analogous to Equations $(1.5)$, the wavevector $(\bar{p}=\nabla \phi)$ and frequency $\left(\omega=-\frac{\partial \phi}{\partial t}\right)$ have been introduced. The coefficient of $(i \lambda)^{2}$ term is seen to be Maslov's Hamiltonian

$$
H=\bar{p} \cdot \ddot{p}-f(\bar{r}, t)_{\omega}^{2}+g(\bar{r}, t) .
$$

The field at any space-time point $(\bar{r}, t)$ proceeds from the stationary phase $\quad\left(\nabla_{p}=0\right)$ evaluation of the integral in Equation (1.2), which turns the Hamiltonian into an eikonal equation

$$
\bar{p} \cdot \bar{p}-f(\bar{r}, t)_{0}^{2}+g(\bar{r}, t)=0
$$

and determines the time-parameterized Lagrange Manifold

$$
\overline{\mathbf{r}}=\nabla_{\mathrm{p}} s(\overline{\mathrm{p}}, \mathrm{t}) \text {. }
$$

In the Lagrange Manifold formalism caustic points are determined from the phase [5], or equivalently from $s(\bar{p}, t)$. To obtain this $s(\bar{p}, t)$, we first find the trajectories (through Hamilton's equations)

$$
\begin{array}{ll}
\bar{r}=\bar{r}(r, \bar{\sigma}) & \bar{p}=\bar{p}(r, \bar{\sigma}) \\
t=t(r, \bar{\sigma}) & \omega=\omega(r, \bar{\sigma})
\end{array}
$$

then invert the wavevector and time transformations

$$
\bar{p}=\bar{p}(r, \bar{\sigma}) \quad t=t(r, \bar{\sigma})
$$

to obtain

$$
r=r(\bar{p}, t) \quad \bar{\sigma}=\bar{\sigma}(\bar{p}, t) .
$$

Substitution into the coordinate space map determines the Lagrange Manifold explicitly

$$
\overline{\mathbf{r}}=\overline{\mathbf{r}}(r(\bar{p}, t), \bar{\sigma}(\bar{p}, t))=\nabla_{p} s(\bar{p}, t),
$$

where the time appears as a parameter. Then an integration along the trajectories

$$
S(\bar{p}, t)=\int_{\bar{p}_{0}}^{\bar{p}} \overline{\mathbf{r}} \cdot d \bar{p}
$$

leads to the phase

$$
\phi(\overline{\mathbf{r}}, \overline{\mathbf{p}}, t)=\overline{\mathbf{r}} \cdot \overline{\mathbf{p}}-S(\overline{\mathbf{p}}, t) \text {. }
$$

The caustic points, equivalent to those specified in Equation $(1.10)$, are those at which 


$$
\operatorname{det}\left\{\frac{\partial^{2}+}{\partial p_{i} \partial p_{j}}\right\}=\operatorname{det}\left\{\frac{\partial^{2} s}{\partial p_{i} \partial p_{j}}\right\}=0 \quad ;(i, j=1,2,3)
$$

At a given time this condition leads to sets of triplets $(\bar{p})$, which upon substitution into Equation (2.5) determines the caustic in coordinate space. The time evolution of the caustic proceeds by considering Equation (2.10) for several values of time. Corresponding to each such time is a set of triplets $(\bar{p})$, which upon substitution into the Lagrange manifold yield the time evolution of the caustic in coordinate space.

The determination of the field on the caustic requires the development of a transport equation for the amplitudes. As this development so parallels that referred to above [5], we do not include it here.

\section{EXAMPLE}

As an example, we consider wave propagation in a medium with $\mathbf{f}(\overline{\mathbf{r}}, t)=1, \quad \boldsymbol{g}(\overline{\mathbf{r}}, t)=b x+a t-k^{2}$, with $a, b, k$ constants. Consequently, the wave equation we consider is

$$
\nabla^{2}-\frac{\partial^{2} \psi}{\partial t^{2}}-\lambda^{2}\left(b x+a t-k^{2}\right)=0 .
$$

Let the initial-boundary condition be that at $\gamma=0$, a point source at the origin $\bar{r}=(0,0)$ begins radiating at $t=t_{0}$, with initial frequency $a$ and wave vector $\bar{p}=\left(p_{\circ} \cos \theta, p_{0} \sin \theta\right)$. We assume an asymptotic solution of the form

$\varphi(\bar{r}, t)-\int A(\bar{r}, \bar{p}, t, \lambda) \exp \{i \lambda(\bar{r} \cdot \bar{p}-s(\bar{p}, t))\} d \bar{p}=0\left(\lambda^{-\infty}\right)$

Then proceeding through the algorithm we obtain Maslov's Hamiltonian (Equation (3.3)) and eikonal (Equation (3.4)), respectively

$$
\begin{aligned}
& H=\bar{p} \cdot \bar{p}-\omega^{2}+b x+a t-k^{2} \\
& \bar{p} \cdot \bar{p}-\omega^{2}+b x+a t-k^{2}=0 .
\end{aligned}
$$

Next Hamilton's Equations (1.6) and (1.7), together with the initial conditions at $r=0$, are solved to obtain the maps

$$
\begin{array}{ll}
x=b r^{2}+2 p_{0} r \cos \theta & p_{x}=-b r+p_{0} \cos \theta \\
y=2 p_{0} r \sin \theta & p_{y}=p_{0} \sin \theta \\
t=a r^{2}+2 r \Omega+t_{0} & v=a r+a .
\end{array}
$$

While Hamilton's Equations relate the canonical variables, the selection of the initial specific condition $t=t_{0}$ at $r=0$ introduces an additional coupling between $t_{0}$ and $\&$ from the eikonal equation

$$
p_{o}^{2}-a^{2}-k^{2}+a t_{0}=0 .
$$

It is this coupling which allows the inclusion of time $t$ as a parameter in the Lagrange Manifold. Specifically, elimination of $t_{0}$ between the time coordinate in Equation (3.7) and Equation (3.8), allows the arc length $r$ to be parameterized in time $t$. Then, inversion of the map $\bar{\mu} \rightarrow \bar{p}$ includes the time as a parameter, which is subsequently introduced into the Lasrange manifold. To illustrate, for clarity of exposition, we first use the simplicity of the example to eliminate $\theta$ between 
Equations (3.5) and (3.6), obtaining

$$
\begin{aligned}
& \mathrm{x}=b r^{2}+2 r p_{\mathrm{x}} \\
& \mathrm{y}=2 r p_{\mathrm{y}}
\end{aligned}
$$

Then from the space and time coordinates in Equations $(3.5)-(3.7)$ and Equation (3.8), we determine

$r=r\left(p_{x}, p_{y}, t\right)=\frac{\left(b p_{x}-a s\right) \neq\left[\left(a \Omega-b p_{x}\right)^{2}-\left(a^{2}-b^{2}\right)\left(a^{2}+k^{2}-p_{x}^{2}-p_{y}^{2}-a t\right)\right]^{1 / 2}}{\left[a^{2}-b^{2}\right]}$

Finally, substituting into Equations (3.9) and (3.10), followed by integrations with respect to $p_{x}$ and $p_{y}$ respectively leads to the phase

$$
\begin{aligned}
\phi(\bar{r}, \bar{p}, t)=\vec{r} \cdot \vec{p}-\frac{2 a}{\left(b^{2}-a^{2}\right)^{2}}\left[a b\left[a^{2} p_{x}+\frac{p_{x}^{3}}{3}\right]-\frac{\left[b^{2}+a^{2}\right] 2 p_{x}^{2}}{2}\right]+ \\
+\frac{2}{3\left(b^{2}-a^{2}\right)^{2}}\left[\left(a a-b p_{x}\right)^{2}-\left(a^{2}-b^{2}\right)\left(a^{2}+k^{2}-p_{x}^{2}-p_{y}^{2}-a t\right)\right]^{3 / 2} \\
\quad-\frac{1}{b^{2}-a^{2}}\left[b\left(a^{2}+k^{2}-p_{y}^{2}-a t\right) p_{x}-\frac{b p_{x}^{3}}{3}+a o p_{y}^{2}+b^{2} a t\right] .
\end{aligned}
$$

(We note only the minus sign in Equation (3.11) leads to physically realizable caustics.)

As a specific example to illustrate the calculational aspects of the alsorithm, let us choose $a=-1, b=6.655\left(=45 / 7 \cos 15^{\circ}\right), k^{2}=23, \theta=15^{\circ}, t_{0}=1, a=5$, $\left|\bar{p}_{0}\right|=7$ and the initial amplitude at the emitter $A_{0}=1$. For this case the Lagrange Manifold and phase are, respectively,

$x=7.556+.154 t+.242 p_{x}-.147 p_{x}^{2}-.154 p_{y}^{2}-\left(0.046 p_{x}+1.537\right) \cdot$

$\cdot\left(1.122+0.231 t+.0355 p_{x}+.0005 p_{x}{ }^{2}-.0231 p_{y}^{2}\right)^{1 / 2}$

$y=-p_{y}\left[2.232+.308 p_{x}+2\left(1.122+.0231 t+.0355 p_{x}+.005 p_{x}^{2}-.0231 p_{y}^{2}\right)\right]$

An integration leads to the phase

$$
\begin{aligned}
& \phi(\bar{r}, \bar{p}, t)=\bar{r} \cdot \bar{p}-5.116 t-7.556 p_{x}-0.121 p_{x}^{2}+.116 p_{y}{ }^{2}-.154 t p_{x} \\
& +.049 p_{x}^{3}+.154 p_{x} p_{y}^{2}+28.86\left[1.122+.0355 p_{x}+.0231 t+.0005 p_{x}{ }^{2}-.0231 p_{y}^{2}\right]^{3 / 2}
\end{aligned}
$$

Then at $r=1$, i.e., the space-time point $(x, y, t)=(6.87,3.62,10$.$) , the classical$ map becomes sinsular, as does the Hessian determinant of the phase (Equation $(2.10)$ ) at the corresponding point in wavevector space $\bar{p}=(.106,1.812)$. This illustrates the level-equivalence of the classical map and the transformation specified by the Lagrange Manifold. To find the caustic at $t=10$, we find those sets of wavevectors $(\bar{p})$ satisfying Equation $(2.10)$; substituting these wavevectors 
into the Lagrange Manifold determines the caustic. The time evolution of the caustic proceeds by substituting successive values of time into the phase and repeating the procedure, (Figure 1).

We note that even over lons time duration, the topological type of this caustic does not change, cf. Mather[6]. The determination of the field at the caustic proceeds largely as in [5]. For completeness, we note that at $(x, y, t)=(6.87$, $3.62,10$.$) , the first two terms in the asymptotic series are$ $\psi(6.87,3.62,10)=.\exp \{i \tau(\pi / 4+9.8)\} x^{1 / 2}\left[-1.53 \Gamma\left[\frac{1}{3}\right] \tau^{-5 / 6} \cos \frac{\pi}{6}-1.74 \Gamma\left[\frac{2}{3}\right] \tau^{-7 / 8} \sin \frac{1}{3}\right]$

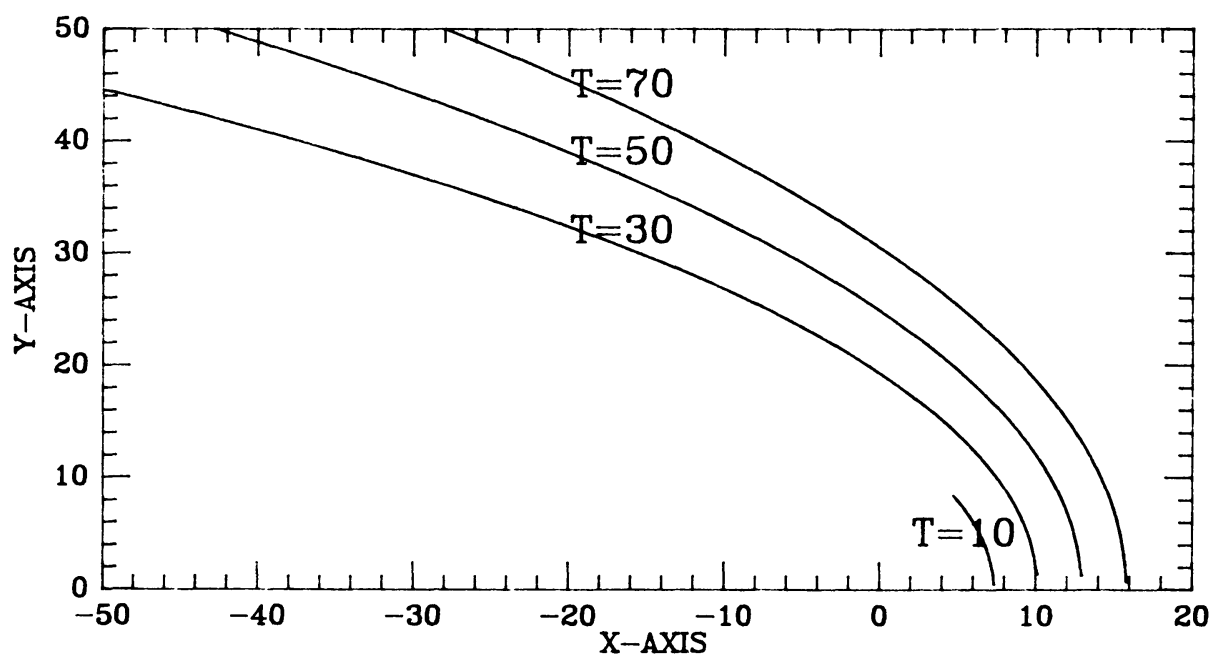

TIME EVOLUTION OF A CAUSTIC

FIGURE 1.

Acrnowlubctarant. One of us (A.D.G.) wishes to gratefully acknowledge helpful discussions with R. T. Prosser and the partial support of NSF grant DNS-8409392.

\section{RASFERdonces}

1. KEILLR, J. B. in CALCULUS OF VARIATIONS AND ITS APPLICATTONS (McGrawHill, New York, 1958).

2. LEWIS, R. M. Asymptotic Theory of Wave Propagation, Arch. Rat. Mech. Anal., 20 (1965), 191-250.

3. MASLOV, V. P. THBORIB DES PERTURBATIONES ET METHODES ASYMPTOTIQUES (Dumod, Gauthier-Villars, Paris, 1972).

4. ARNOLD, V. I. Characteristic Class Entering in Quantization Conditions, Funct. Anal. Appl., 1 (1967), 1-13.

5. GORMAN, A. D. Space-Time Caustics, Internat. I. Math. Math. Sci. $\underline{9}$ (1986), 531-540.

6. MATHER, J. Stability of $\mathrm{C}^{\infty}$ - Mappings II, Infinitesimal Stability Implies Stabilit, Ann. Math., 89 (1969), 254-291. 


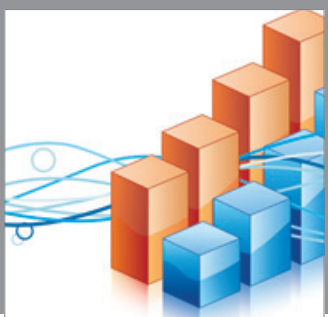

Advances in

Operations Research

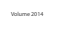

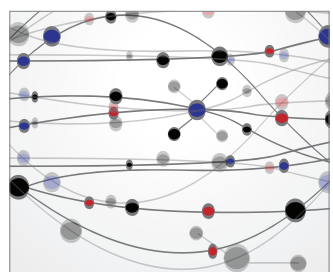

\section{The Scientific} World Journal
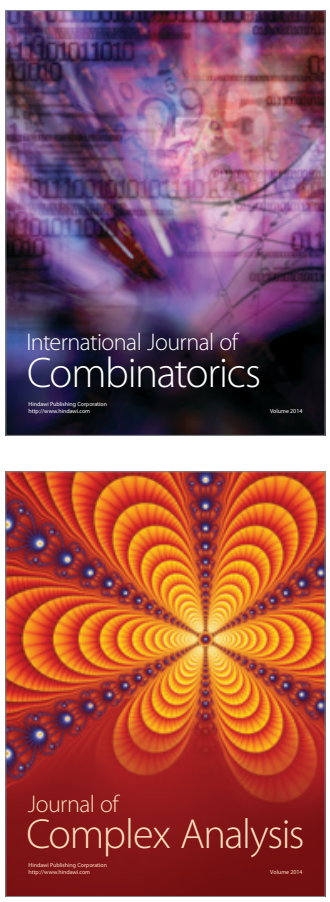

International Journal of

Mathematics and

Mathematical

Sciences
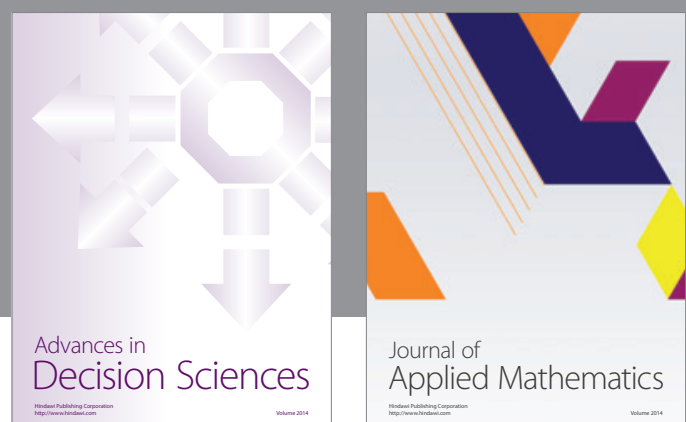

Journal of

Applied Mathematics
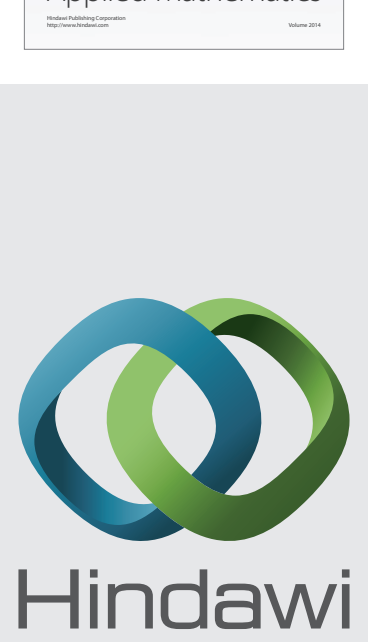

Submit your manuscripts at http://www.hindawi.com
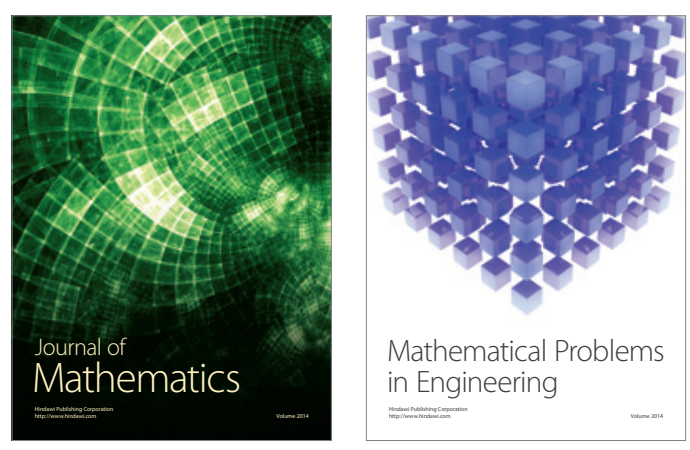

Mathematical Problems in Engineering
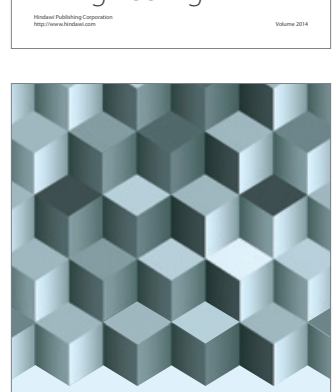

Journal of

Function Spaces
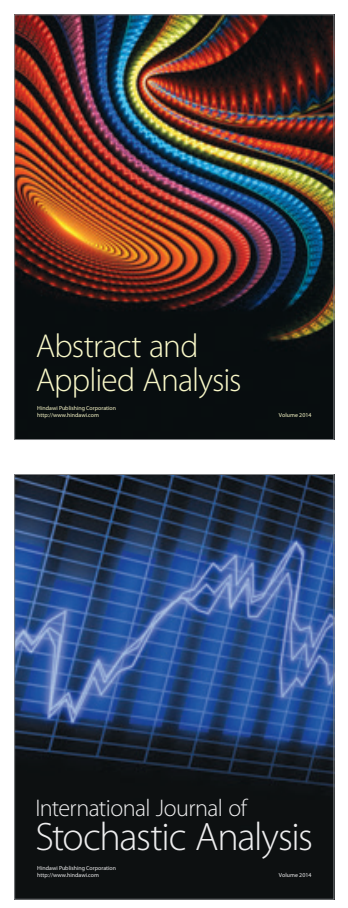

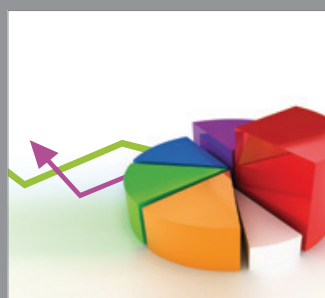

ournal of

Probability and Statistics

Promensencen
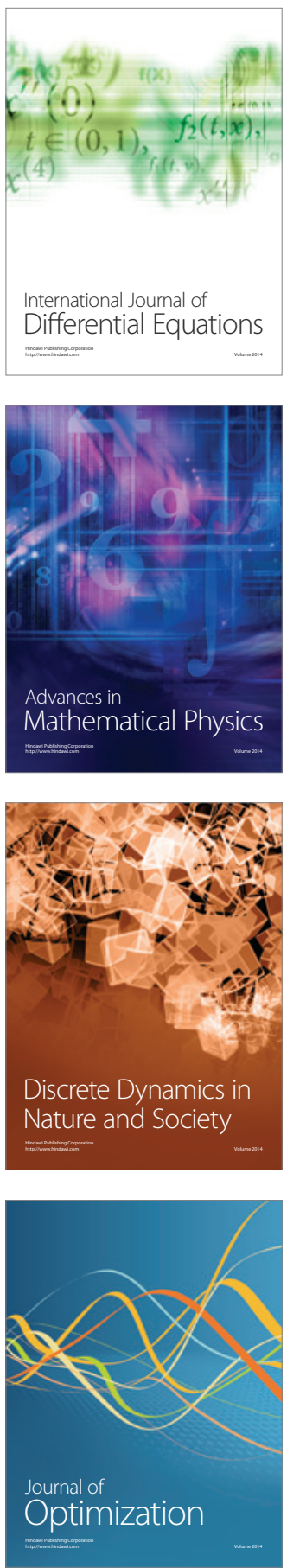